\title{
Matrices et quadripôles, une passerelle entre les mathématiques et l'électronique
}

\author{
Norbert Verdier, Antoine Diet, Souhil Megherbi \\ norbert.verdier@u-psud.fr, antoine.diet@u-psud.fr, souhil.megherbi@u-psud.fr \\ IUT de CACHAN, département de Génie Electrique et Informatique Industrielle 1 (Geii-1) \\ $\&$ \\ Adrien Larbanet et Enguerrand Marcellin, étudiants à l'IUT de Cachan. \\ 9, avenue de la division Leclerc BP 14094234 CACHAN
}

RESUME : Depuis quelques années l'algèbre linéaire et plus particulièrement l'étude des matrices, n'est plus enseignée à l'IUT de Cachan (Geii1). Ce cours maniant différents types d'objets à des niveaux divers (fonctions, vecteurs, bases, matrices, etc.) présentaient des difficultés de compréhension à notre public de DUT. Nous avons décidé de réintroduire cette discipline à une partie de la promotion en la corrélant à l'étude d'un problème issu de l'électronique (étude des quadripôles). L'objectif était de présenter la «théorie des matrices » en se restreignant au cas des matrices $(2,2)$ pour sa force opératoire et pratique. Les éléments de mathématiques et les applications à l'électronique ont été présentés parallèlement « pour le meilleur et pour le pire ».

\section{1) Introduction}

Ce cours (ou module) complémentaire alliant mathématiques et électronique a été proposé en début du semestre 2 du DUT-Geii. Il fait partie du pôle intitulé «Apprendre autrement ${ }^{1}$. Il a fait l'objet d'un module destiné à préparer à une poursuite d'études en écoles d'ingénieurs. Sur la centaine d'étudiants composant la promotion, 18 se sont inscrits. Certains présentaient un très bon dossier universitaire (les premiers de promotion), quelques-uns s'étaient inscrits malgré un niveau scientifique « insuffisant». La partie mathématique était composée de quatre cours de deux heures. Elle avait pour finalité d'enseigner les éléments d'algèbre linéaire afin de pouvoir comprendre les applications à l'étude des quadripôles. La partie électronique a été organisée en quatre cours de deux heures intercalés entre les cours de mathématiques, puis une illustration en travaux pratiques. En parallèle, un «devoir à la maison» $(\mathrm{DM})$ de réflexion sur la modélisation simplifiée d'un câble coaxial 50 Ohms, (sans utiliser la théorie des paramètres $\mathrm{S}$ qui ne sera vue qu'en deuxième année), a servi de fil rouge à cet enseignement. En effet ce DM fait appel à la diagonalisation et aux produits de matrices, notions directement issues du cours de mathématiques.

\footnotetext{
${ }^{1}$ Les autres cours proposés à l'IUT de Cachan sont de nature diverse (formules de remise à niveau en mathématiques ou en Anglais, apprentissage d'un logiciel de calcul formel, spécialisation dans un des domaines de l'électronique, etc.). Chaque étudiant doit bien entendu être inscrit à un de ces cours du module "Apprendre autrement ». Les inscriptions relèvent le plus souvent du choix de l'étudiant, parfois ils sont "suggérés » par l'équipe enseignante. Le module présenté ici n'a été "imposé» à aucun étudiant; il a seulement été présenté à l'ensemble de la promotion et conseillé à certains étudiants visant une poursuite d'études dans des écoles d'ingénieurs « classiques et sélectives ».
}

2) « Tout sur les matrices à 2 lignes, 2 colonnes et en 8 heures!"

\section{Qu'est-ce qu'une matrice? Une matrice inverse?}

La première leçon avait pour finalité de répondre à la question: Qu'est-ce qu'une matrice? Les étudiants savaient résoudre les systèmes d'équations linéaires. Partant de là, il n'a pas été difficile d'introduire une matrice de la forme visée ( 2 lignes et 2 colonnes) sous la forme d'un tableau à 4 éléments. Un système linéaire peut ainsi se modéliser sous la forme : $Y=A$. $X$ où Y et $\mathrm{X}$ sont des vecteurs ayant 2 coordonnées et où $\mathrm{A}$ est la matrice associée au système en question. En résolvant le système, on aboutit ainsi à la matrice inverse qu'on note $\mathrm{A}^{-1}$ par analogie à une analyse classique (les étudiants avaient vu dans le cours du semestre 1 les bases de l'analyse élémentaire et connaissaient donc la notion de fonction inverse). Cette première leçon a été illustrée par plusieurs exercices numériques où il était demandé de trouver la matrice modélisant telle ou telle situation et d'inverser ensuite la matrice trouvée. Nous avons introduit la notion de déterminant et insisté sur son utilisation principale : une matrice est inversible si et seulement si son déterminant est non nul ce qui revient à dire qu'un système admet une unique solution si et seulement si le déterminant associé est non nul.

\section{Opérations matricielles}

La deuxième leçon avait pour ambition de montrer les limites de la première définition d'une matrice, réduisant cet objet à n'être qu'un tableau de nombres. Nous avons développé la signification profonde d'une matrice: une matrice est la représentation d'une application linéaire dans une certaine base ${ }^{2}$. C'est cette définition qui permet de construire les opérations

\footnotetext{
${ }^{2}$ Nous avons défini une base du plan comme étant l'ensemble de deux vecteurs (non colinéaires) $\mathbf{u}$ et $\mathbf{v}$. Ces deux vecteurs permettent de « reconstituer » tous les autres vecteurs du plan.
} 
algébriques matricielles: additions, soustractions et multiplications. Nous avons fortement insisté sur cet aspect et effectué (sur des exemples numériques) de nombreuses opérations matricielles. La séance s'est terminée sur l'itération matricielle : une matrice $A$ étant donnée, comment calculer $A^{n}$ ? Avant de se pencher sur des exemples, nous avons montré pourquoi ce type de calcul est crucial dans l'étude des circuits. Nous avons fait calculer $A^{n}$ dans des cas «simples »: $A$ est diagonale et $A$ s'écrit sous la forme $\left(\begin{array}{ll}\lambda & 1 \\ 0 & \lambda\end{array}\right)$. Pour cela, il suffit de multiplier la matrice par elle-même afin de « proposer » une formule générale « à l'ordre $n$ » qui se démontre par récurrence sur $n$. Dans le « cas général», en prenant un exemple de matrice quelconque, nous avons fait sentir que ce problème est difficile et que la méthode «frontale » (multiplier par elle-même la matrice et «deviner» la formule générale de $A^{n}$ ) conduit en général à l'échec.

\section{Effets d'un changement de base}

La troisième leçon se proposait d'introduire la notion de changement de base afin d'être capable d'élever à la puissance $n$ n'importe quelle matrice. Pour ce faire nous avons commencé à démontrer quel était l'effet d'un changement de base sur un vecteur et sur une application linéaire. Nous avons ainsi introduit la notion de matrice de passage $P$ d'une base à une autre et avons démontré les deux formules fondamentales :

$$
\text { (1) } X=P X^{\prime}
$$

où $X$ représente un vecteur exprimé dans une « ancienne » base et où $X$ ' représente le même vecteur exprimé dans la «nouvelle» base.

$$
\text { et (2) } \quad A=P^{-1} B P
$$

où $B$ est la matrice de $f$ dans la « nouvelle » base et où $A$ est la matrice de $f$ dans une « ancienne » base.

Nous avons effectué des applications « numériques » et avons déduit de (2) que $A^{n}=P^{-1} B^{n}$ $P$. Nous avons insisté sur la « force» de cette dernière expression: si nous sommes capables de trouver une « nouvelle» base dans laquelle $B$ est « simple» (diagonale par exemple) alors de facto nous serons capable de déduire $A^{n}$.

\section{Diagonaliser et trigonaliser}

La quatrième leçon avait pour objectif d'apprendre à diagonaliser et trigonaliser une matrice. Nous avons commencé par introduire la notion de valeur propre. Pour diagonaliser, nous devons trouver un vecteur $\mathrm{X}$ (non nul) tels que : $\mathrm{A} . \mathrm{X}=\lambda \mathrm{X}$. Par linéarité, tous les vecteurs multiples de $X$ vérifient le système : le système a donc une infinité de solutions ce qui revient à dire grâce à la notion de déterminant : det
$(\mathrm{A}-\lambda \mathrm{I})=0$; cette quantité est appelée polynôme caractéristique de A. Si la matrice est donnée par: $\mathrm{A}=\left(\begin{array}{ll}a & b \\ c & d\end{array}\right)$ alors $\operatorname{det}(\mathrm{A}-\lambda \mathrm{I})=\lambda^{2}-\lambda(a+b)+\operatorname{det} A$.

C'est ici un polynôme du deuxième degré. Ses racines sont appelées "valeurs propres» de $A$. On appelle espace propre associé à une valeur propre $\lambda$ l'ensemble des vecteurs $X$ du plan tel que : A.X $=\lambda X$. On note cet espace $\mathrm{E}(\lambda)$. Si $\lambda$ est une valeur propre simple (c'est-àdire une racine simple du polynôme caractéristique) $\mathrm{E}(\lambda)$ est une droite; si elle est double, c'est une droite ou un plan. Il en résulte que nous devons dissocier le cas où il $\mathrm{y}$ a deux valeurs propres distinctes du cas où il n'a qu'une valeur propre double.

a) Quand la matrice admet deux valeurs propres simples

Nous avons déduit la situation générale de l'examen d'un cas particulier : $A=\left(\begin{array}{ll}1 & 1 \\ 1 & 1\end{array}\right)$. Soient $\lambda_{1}$ et $\lambda_{2}$ les deux valeurs propres. Dans ce cas, $\mathrm{E}\left(\lambda_{1}\right)$ est une droite de vecteur directeur $\vec{u}$ et $\mathrm{E}\left(\lambda_{2}\right)$ est une droite de vecteur directeur $\vec{v}$. Dans cette «nouvelle» base $\{\vec{u}$, $\vec{v}\}$, l'application linéaire associée a pour matrice :

$\mathrm{B}=\left(\begin{array}{cc}\lambda_{1} & 0 \\ 0 & \lambda_{2}\end{array}\right) \quad$ : nous avons diagonalisé la matrice

Il est ensuite aisé de calculer $B^{n}$ et $A^{n}$ (grâce à la formule reliant $A$ à $B$ via le changement de base : $B=$ $\left.P^{-1} A P\right)$. [Cf. Troisième leçon]

b) Quand la matrice admet une valeur propre double

Si la matrice admet une valeur propre double $\lambda$. Dans ce cas, $E(\lambda)$ est soit une droite soit le plan comme nous l'annoncions précédemment. Si c'est le plan, il est aisé de démontrer que $B=A=\lambda I$. C'est un cas trivial. Sinon, nous avons illustré l'étude avec $A=\left(\begin{array}{cc}3 & -1 \\ 1 & 1\end{array}\right)$ pour décrire la situation générale : si $E(\lambda)$ est une droite de vecteur directeur $\vec{u}$. On démontre qu'on peut toujours construire une base $\{\vec{u}, \vec{v}\}$ avec $\vec{v}$ tel que $f(\vec{v})=\vec{u}+\lambda \vec{v}$. Dans cette «nouvelle» base, la matrice de $f$ est : $B=\left(\begin{array}{ll}\lambda & 1 \\ 0 & \lambda\end{array}\right)$

C'est une matrice triangulaire. On dit qu'on a « trigonalisé » la matrice sous une forme particulière 
appelée «forme de $\operatorname{Jordan}^{3}$ ». Le calcul de $B^{n}$ ne pose pas de problème. Le calcul de $A^{n}$ se déduit de la formule : $B=P^{-1} A P$.

La fin du cours de mathématiques coïncidait avec la remise, dans le cours d'électronique, d'un formulaire dont voici un extrait présentant la situation dans le cas où la matrice a deux valeurs propres simples :

$$
\begin{aligned}
& \left(\begin{array}{ll}
\mathrm{A} & \mathrm{B} \\
\mathrm{C} & \mathrm{D}
\end{array}\right)^{\mathrm{n}}=\underline{\underline{\mathrm{P}}}\left(\begin{array}{cc}
\lambda_{1}^{\mathrm{n}} & 0 \\
0 & \lambda_{2}^{\mathrm{n}}
\end{array}\right) \underline{\mathrm{P}}^{-1} \\
& =\frac{1}{v-u}\left(\begin{array}{ll}
1 & 1 \\
u & v
\end{array}\right)\left(\begin{array}{cc}
\lambda_{1}^{n} & 0 \\
0 & \lambda_{2}^{n}
\end{array}\right)\left(\begin{array}{cc}
v & -1 \\
-u & 1
\end{array}\right) \\
& =\frac{1}{v-u}\left(\begin{array}{cc}
v \lambda_{1}^{n}-u \lambda_{2}^{n} & \lambda_{2}^{n}-\lambda_{1}^{n} \\
u v\left(\lambda_{1}^{n}-\lambda_{2}^{n}\right) & v \lambda_{2}^{n}-u \lambda_{1}^{n}
\end{array}\right)
\end{aligned}
$$

L'ambition du cours de mathématiques était d'amener les étudiants à comprendre le concept fondamental (changement de base) qui permettait l'utilisation à venir de ces formules. Nous avons clôt le cours en signalant que tout ce qui avait été développé dans ce cours pour des matrices à deux lignes et deux colonnes se généralisait à des matrices à $n$ lignes et $n$ colonnes [1]. Pour une application des matrices (cas général) à l'électronique, nous avons renvoyé à l'article rédigé par Patrick Lagonotte et Yves Eichenlaub [2] ainsi qu'au serveur d'exercices WIMS (développé en partie à l'Université Paris-Sud) pour apprendre à manipuler les calculs matriciels de manière interactive et quasi - ludique [http://wims.auto.u-psud.fr/wims/].

\section{3) A quoi servent les matrices en électronique ?}

Les matrices sont considérées, à juste titre, comme des objets mathématiques qui vont aider l'électronicien pragmatique à analyser un circuit électrique. La première étape du raisonnement consiste à amener les étudiants à considérer les matrices comme une description synthétique des relations électriques linéaires (« linéarisées » pour les quadripôles actifs) entre les courants et les tensions d'entrées et de sorties d'un circuit. Dans le cours présent le circuit est considéré avoir deux ports, d'où la restriction à des matrices $2 \times 2$. La vision électronique du cours consiste donc à expliquer l'intérêt d'utiliser les matrices pour décrire le lien entre le signal d'entrée $\left(\mathrm{V}_{1}, \mathrm{I}_{1}\right)$ et le signal de sortie $\left(\mathrm{V}_{2}, \mathrm{I}_{2}\right)$ d'un circuit ou d'un souscircuit (l'intérêt du sous-circuit apparaissant lors des associations élémentaires de quadripôles). Dans la

\footnotetext{
3 Pour presque tout savoir sur Jordan et son théorème, nous renvoyons à la thèse de Frédéric Brechenmacher: "Histoire du théorème de Jordan de la décomposition matricielle (1870-1930) », thèse EHESS, 2006.

Cf.http://fredericbrechenmacher.noosblog.fr/frdric brechenmacher/th ese de doctorat/index.html.
}

partie consacrée à la diagonalisation des matrices chaines pour la mise en cascade de quadripôles, le cas général est celui où la matrice possède 2 valeurs propres. Les deux cas particuliers de l'impédance série et de l'impédance parallèle sont également présentés ${ }^{4}$. Les supports de cours, le sujet de TP et le sujet du DM sont sous la plateforme DOKEOS de l'Université Paris-Sud 11 [4].

\section{Prendre du recul par rapport à un schéma}

Le cours s'adresse à des étudiants ayant déjà validé un semestre du cursus Geii dans lequel sont présentées les notions de dipôles linéaires $\mathrm{R}$, $\mathrm{L}$ et $\mathrm{C}$, leurs associations, les sources équivalentes de Thévenin et de Norton, le théorème de Millmann et les diagrammes de Bode. En nous appuyant sur ces acquis, nous posons un premier problème d'analyse classique : la recherche d'une impédance équivalente entre un point du circuit ( $\mathrm{A} »$, sur la figure 1) et la masse. Dans le cadre de la détermination des résistances équivalentes de Thévenin et de Norton, cela parait d'un intérêt évident. Soumettons maintenant aux étudiants le schéma suivant et laissons les analyser le problème en trois temps: (1) Nous ne donnons pas de pistes de calcul dans un premier temps.
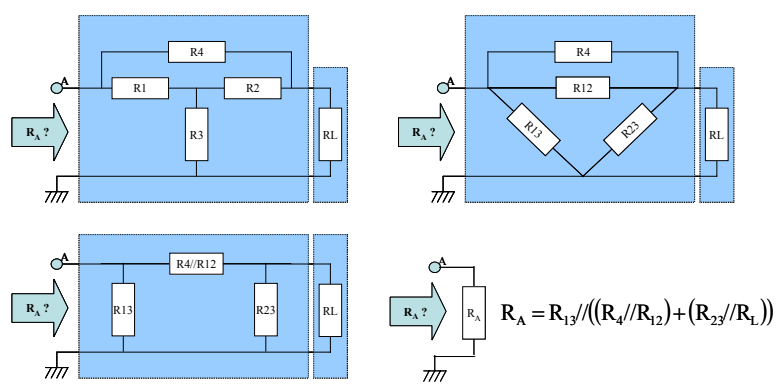

figure 1 : utilisation de la transformation

Compte tenu des acquis théoriques, le calcul classique d'une impédance équivalente sur un quadripôle en «Té ponté » est long et fastidieux. (2) Donnons leurs un premier outil de calcul : la transformation étoile/triangle. Il apparait alors une résolution du problème plus rapide après une étape de transformation du circuit, voir la figure 1. Cependant cette solution est à critiquer car elle présente l'inconvénient de modifier la topologie électrique du circuit. En effet, le nœud «A » disparait par la transformation étoile/triangle. (3) Orientons leur réflexion sur l'importance d'une solution modulaire dont les paramètres sont aisément évolutifs et qui ne modifie en rien la topologie analysée, mais seulement notre vision du problème : prenons du recul. C'est la

\footnotetext{
${ }^{4}$ Dans le cas des quadripôles passifs, les matrices chaines qui possèdent une valeur propre double sont l'impédance série seule et de l'impédance parallèle seule. Ces matrices chaînes sont sous forme triangulaires (le lien avec la forme trigonal est alors immédiat). Nous n'abordons pas dans ce cours la mise en cascade de quadripôles actifs particuliers (valeur propre double).
} 
solution présentée sur la figure 2. Le calcul de la solution a été déroulé de manière succincte afin de commencer à familiariser les étudiants avec l'association du terme matrice à un circuit ou à une partie du circuit (matrice du sous-circuit). L'intérêt de l'outil ayant été abordé, il nous faut alors apporter le formalisme nécessaire à la mise en équation matricielle et les règles de maniement de ces objets : propriétés mathématiques et électriques (il $\mathrm{y}$ a souvent une implication réciproque) et lois d'associations élémentaires (que signifie une somme ou un produit matriciel en électronique?).

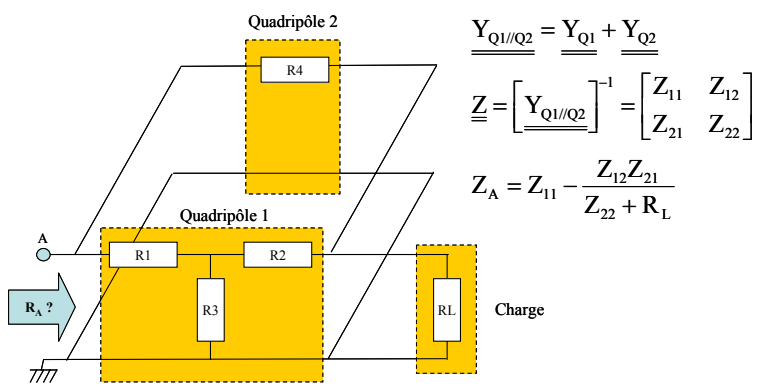

figure 2 : utilisation des quadripôles

Nous insistons enfin sur un dernier point courant en électronique : comprendre et mettre en équation l'influence d'un circuit sur un autre lors de la mise en cascade de dispositifs. Par exemple: la source équivalente de Thévenin du générateur est connectée par un câble à un (ou plusieurs !) filtre(s) cascadé(s) et via un autre câble à une impédance de charge. Que devient alors le diagramme de Bode d'un filtre seul dans le cas général? Les cours suivants démontrent qu'un enchainement de multiplications matricielles conduit rapidement à une réponse logique, observables facilement en séances de travaux pratiques.

\section{Des choix à faire sur le contenu du cours}

Compte tenu du nombre d'heures et de sa structure hybride, il faut choisir en priorité le contenu le plus apte à illustrer un parallèle entre les mathématiques et l'électronique.

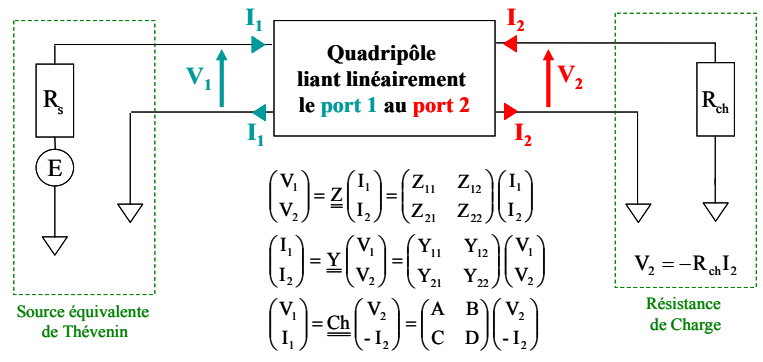

figure 3 : conventions d'écriture des matrices $\underline{\underline{Z}} \underline{\underline{Y}}$ et $\underline{\underline{C h}}$

La théorie des quadripôles est tout d'abord restreinte au cas où l'entrée et la sortie ont la même masse, ce qui est extrêmement fréquent en pratique. Nous pourrions donc parler de tripôles au lieu de quadripôles. Cette différence importante se traduit dans l'obligation de redessiner le schéma électrique d'un quadripôle si le fait de relier les masses vient à courtcircuiter certaines impédances du schéma. L'étudiant distrait qui n'applique pas cette étape de précaution encourt le risque de démontrer à ses frais que le calcul de la matrice du quadripôle puis la mise à $« \mathrm{Z}=0$ » de l'impédance court-circuitée ne conduit pas forcément à la matrice du tripôle... Ceci est un dû à l'application de la loi des nœuds. Ce problème est également présent lors de l'association en série de deux quadripôles qui n'est possible que si le test de Brune est vérifié, sujet que nous n'aborderons pas dans cet article par souci de concision, des informations complètes sont présentes dans le cours de M. Dutoit [3]. Le modèle du tripôle étudié en cours d'électronique est celui de la figure 3 . Les notions du premier semestre de Geii ont permis à l'étudiant de maitriser les associations d'impédances et les fonctions de transfert. Afin d'étendre dans un premier temps ces/ses notions, le choix est fait de ne présenter que les matrices impédance $\underline{\underline{\mathbf{Z}}}$, admittance $\underline{\underline{\mathbf{Y}}}$ et chaine $\underline{\underline{\mathbf{C h}}}$. Ces dernières font par ailleurs appel à toutes les propriétés de base des matrices : addition des matrices impédances (resp. admittances) lors de l'association de quadripôles en série (resp. parallèle), multiplication des matrices chaines lors de la mise en cascade de quadripôles et aussi détermination des propriétés électriques de réciprocité, symétrie et unilatéralité du circuit par observation des propriétés mathématiques des matrices. Le cœur de cours est axé sur la compréhension de l'influence d'un port sur l'autre : entrée sur la sortie et sortie sur l'entrée et ce même en présence d'une impédance de charge ou de générateur quelconque. On présente donc dans cette optique les associations élémentaires de quadripôles afin d'arriver au synoptique de la figure 4 qui décrit le cas général du test d'un quadripôle entre une source de tension (alimentation continue ou source de signaux alternatifs $50 \mathrm{Ohms}$ ) et une charge (autre chose qu'un oscilloscope ?).

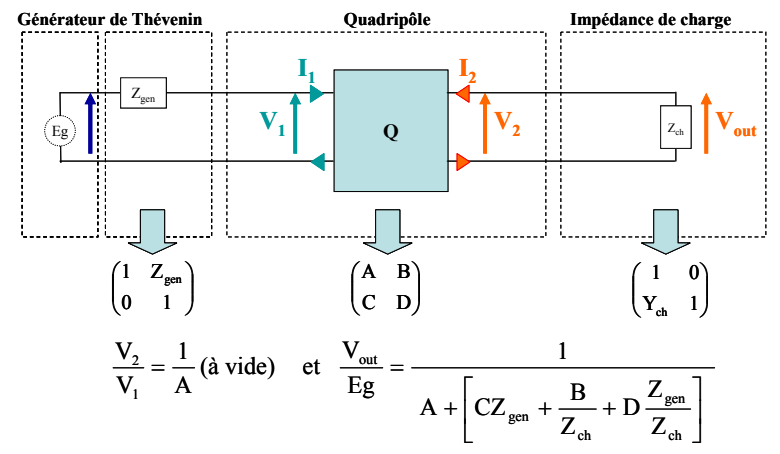

figure 4 : Fonction de transfert " globale " en tension

Le but est alors de faire comprendre l'influence des éléments en cascade les uns sur les autres, notamment lors de la visualisation de la fonction de transfert, étudiée en tension lors du premier semestre 
Geii. La mise en équation est rapide et un test pratique avec des impédances de charges et du générateur identifiées est aisé, ce qui permet de chiffrer la différence entre la fonction de transfert en tension théorique et la fonction de transfert observée entre la tension d'alimentation et la tension aux bornes de la charge (boite à décade). Le parallèle avec la multiplication matricielle permet de mettre en évidence l'influence des éléments de la matrice chaine $\underline{\underline{\mathbf{C h}}}$ qui sont liés aux courants et non plus uniquement aux tensions (B, C et D en l'occurrence). Cette étape du cours permet de sensibiliser nos étudiants aux interactions entre quadripôles en cascade.

\section{De Thévenin/Norton aux schémas équivalents}

Les théorèmes d'équivalence de "Thévenin » (Une source de tension et une résistance en série) et de « Norton » (une source de courant et une résistance en parallèle) sont présentés brièvement aux étudiants du premier semestre de Geii dans le cas restreint des sources non-liées et se ramènent à l'étude d'une maille avec des éléments en série (Les schémas de « Norton » ont leur équivalent en "Thévenin »). La mise en équation matricielle d'un circuit donné sous la forme impédance $\underline{\underline{\mathbf{Z}}}$ ou admittance $\underline{\underline{\mathbf{Y}}}$ est immédiatement illustrée par le schéma équivalent dans lequel apparaissent les quatre paramètres calculés des matrices, la source équivalente de Thévenin et sa résistance en entrée ainsi que l'impédance de charge. Le schéma équivalent le plus facile à illustrer pédagogiquement est celui faisant appel aux éléments de la matrice impédance $\underline{\underline{Z}}$ sur lequel apparaissent clairement deux mailles élémentaires composées d'une source de tension et de deux impédances formant un cas trivial de pont diviseur. La nouveauté apportée dans ce cours est donc d'introduire la notion de source liée comme moyen d'illustrer les relations linéaires au sein d'un quadripôle. La simplicité du schéma équivalent ainsi obtenu permet d'aborder la notion de transfert d'énergie au sein du quadripôle, prélude à un cours de filtrage analogique L-C (comprendre l'adaptation en puissance).

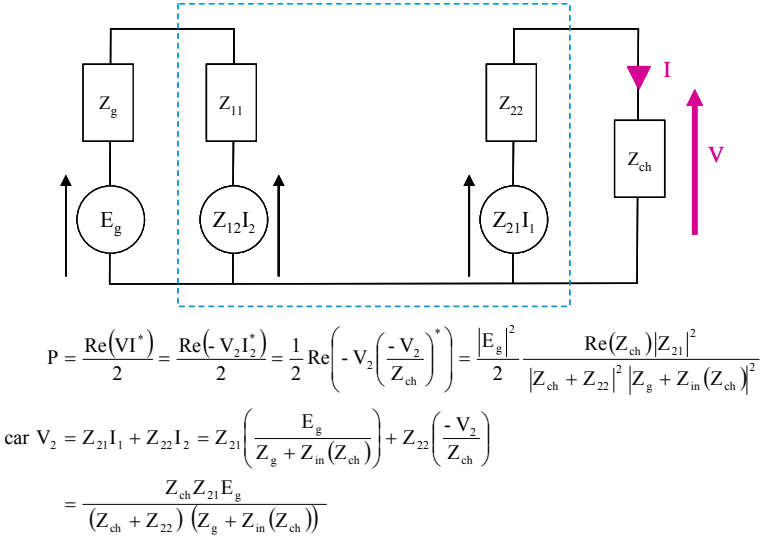

figure 5 : bilan de puissance dans un quadripôle
Un essai a donc été fait à cette occasion de développer les formules de la figure 5 pour démontrer la facilité du calcul en puissance (le bilan de puissance et le filtrage) si la mise en équation matricielle est effectuée dans un premier temps sur un circuit dont le nombre d'éléments n'est pris en compte que lors de la détermination de $\underline{\underline{\mathbf{Z}}}$. La relation existante à l'entrée (resp. la sortie) d'un quadripôle entre le courant et la tension est une relation linéaire qui, après «passage » dans la matrice (il y a alors un sens mathématique donné à cette opération), devient une autre relation présentée sur la sortie (resp. l'entrée). On introduit alors la notion électrique de « charge » d'un port, ce qui entraine la notion d'impédance présentée, en entrée/sortie, par un «quadripôle chargé ». L'illustration la plus parlante étant bien évidemment l'impédance d'entrée du quadripôle en fonction de l'impédance de charge et l'impédance de sortie du quadripôle en fonction de l'impédance du générateur (la source de Thévenin étant de 0 Ohms).
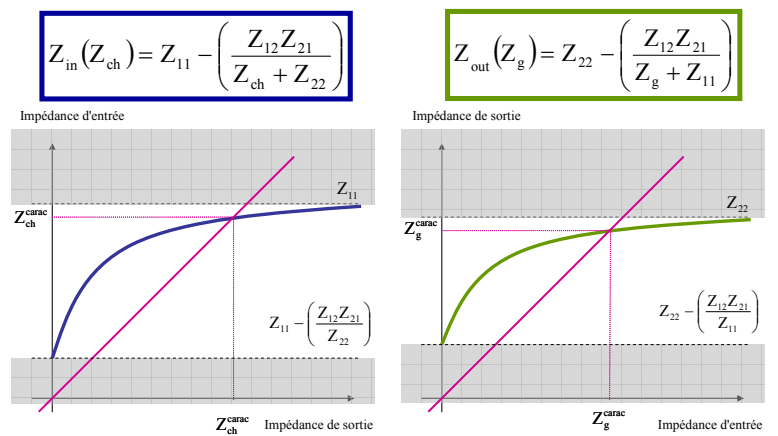

figure 6 : transfert d'impédance due au quadripôle

Il vient alors l'étude des deux fonctions nonlinéaires (parfaitement connues en électronique) dont le tracé est illustré sur la figure 6 dans le cas d'impédance purement résistives, ce qui est fait en travaux pratiques dans un premier temps. Les déterminations des impédances d'entrée et de sortie sont aisément calculables d'après le schéma équivalent de Thévenin de la figure 5. On illustre également le fait que le choix de la matrice pour le positionnement du calcul conduit à la même équation (heureusement) fortement nonlinéaire dont l'étude mathématique impose un rappel de la notion d'asymptote. Il vient alors l'étude des points particuliers et de leurs significations.

\section{L'impédance itérative et la mise en cascade}

D'après l'étude des fonctions impédance d'entrée et impédance de sortie, issues de la transformation par le quadripôle d'une relation linéaire liant un courant à une tension, il existe un point invariant pour lequel l'impédance est présentée d'un port sur l'autre et ce, de manière « itérative »... Ce terme est alors préféré à son synonyme « impédance caractéristique » pour illustrer son importance lors d'une mise en cascade. En effet, la mise en cascade de plusieurs quadripôles d'impédance itérative égale, chargée sur le port concerné par cette 
même impédance, conduit lors de la mise en équation sous la forme chaine $\underline{\underline{\mathbf{C h}}}$ à une multiplication matricielle où chaque produit (itération) conserve cette impédance. L'illustration de ceci se fait aisément en pratique sur des quadripôles et l'élargissement de cette notion est proposée à travers un sujet de réflexion sur le câble coaxial 50 Ohms « à la maison » : un DM dont le schéma est reporté sur la figure 7 . Le but de ce DM est de parvenir à une mise en équation globale mais simplifiée du câble en faisant appel aux produits matriciels des matrices chaines $\underline{\underline{\mathbf{C h}}}$ élémentaires et donc nécessite l'utilisation de la partie du cours de mathématique sur la diagonalisation/trigonalisation. La difficulté est de parvenir à manier des notions de limites car la nature du câble impose une modélisation en termes d'effets inductif et capacitif « répartis » par opposition aux effets inductif et capacitif «localisés » comme ceci est le cas dans une inductance et une capacité de valeur donnée. Il y a donc ici une ouverture supplémentaire de la partie électronique du cours vers un enseignement plus tourné vers la physique. La mise en équation de ces phénomènes physiques nécessitant là aussi un formalisme mathématique précis et rigoureux. Un cours complémentaire de sciences physiques sur cet aspect sera mit en place dans la version suivante du cours en 2009 , ce qui enrichira la transversalité du module d'enseignement.

\section{Discussion et perspectives}

Le cours/TP/DM Mathématiques/Electronique présenté dans cet article a démontré la possibilité de faire interagir des notions nouvelles à la fois en mathématiques et en électronique (voire en physique) autour d'exemples simples. Un simple câble coaxial, manipulé souvent par ces étudiants devient un sujet de réflexion où la mise en équation et la discussion provoquée font appel aux bases théoriques et à leur interprétation. Ce cours est l'occasion de réintroduire dans l'enseignement Geii des parties traditionnellement considérées comme difficilement abordables ou trop théoriques. Nous espérons pouvoir poursuivre cet effort en développant les cas d'études à des problèmes plus larges, nécessitant une étape de recherche et de documentation de la part des étudiants, que cela soit en mathématiques et/ou en électronique (en concertation). Une réflexion pédagogique sur le public étudiant est menée à partir de leur retour et de notre analyse. Il apparait d'après le retour d'un premier groupe d'étudiants testés que le point fort est l'illustration de la complémentarité entre les mathématiques et l'électronique, ce qui était notre premier objectif. Quelques aménagements sur le contenu sont néanmoins nécessaires et l'introduction du thème du $\mathrm{DM}$ en début de module à discuter lors d'une réactualisation de cet enseignement où une courte partie «modélisation des phénomènes physiques » sera introduite (2h).

\section{Bibliographie :}

[1] Belloc, Jean-Claude. 1992, Algèbre (Tome 4), seconde édition, Masson, 1992.

[2] Lagonotte, Patrick \& Eichenlaub, Yves « Réseaux électrocinétiques et algèbre linéaire (notions fondamentales) », J3eA, Vol. 5, 2006. [Cf.http://www.j3ea.org/index.php?option=toc\&url=/articles/j3e a/abs/2006/01/contents/contents.html]

[3] Thierry Dutoit, cours sur la théorie de l'analyse des circuits électriques, chapitre 6 : Quadripôles. http://tcts.fpms.ac.be/cours/1005-01/theocirc6.pdf

[4] Plateforme DOKEOS de l'université Paris-Sud 11 / Science et technologies / A.Diet--Supports de cours // Quadripôles http://formation.u-psud.fr/

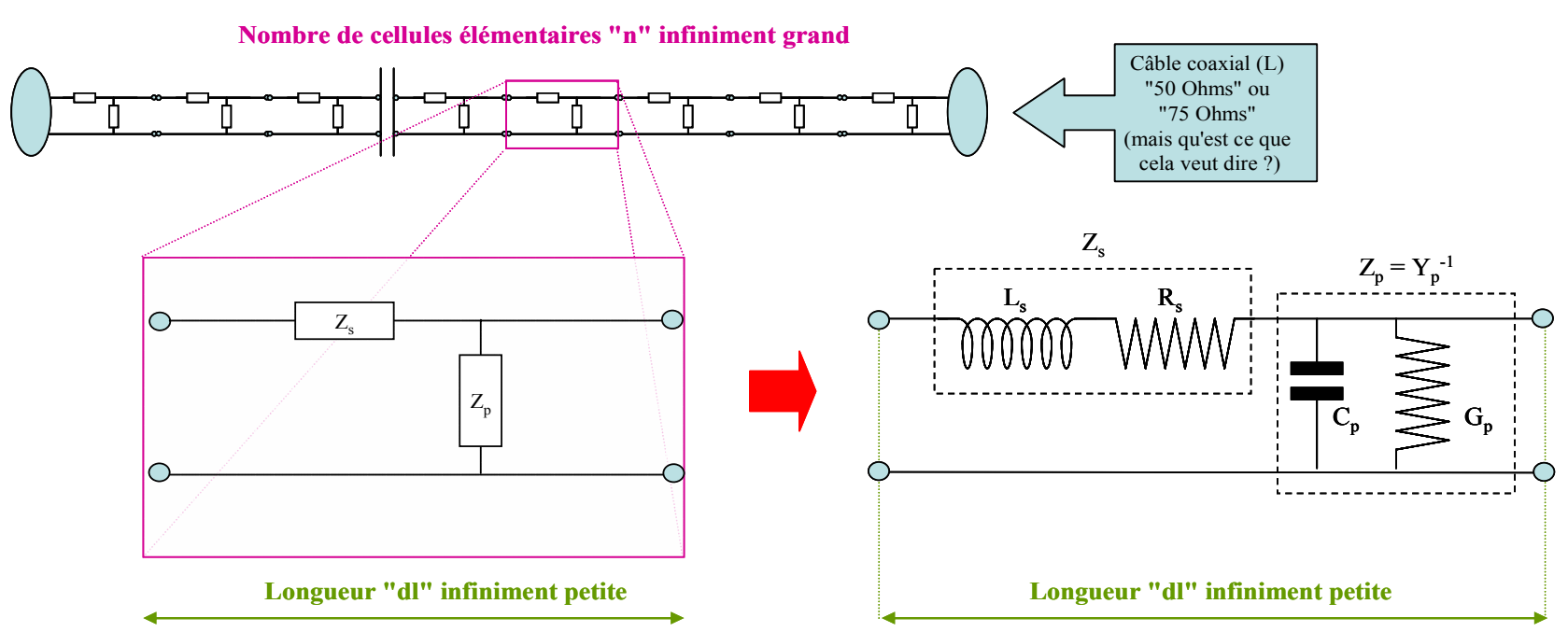

figure 7 : modélisation du câble coaxial 50 Ohms 


\section{Post scriptum : La présentation du poster au CETSIS 2008 à Bruxelles}

Conscients que la participation à un colloque scientifique - lieu d'échanges et de partages au sein d'une même communauté scientifique- est toujours instructive pour comprendre comment les sciences et les techniques se diffusent et se transmettent, nous avons décidé, sur des critères de mérite, d'envoyer au CETSIS 2008 à Bruxelles deux étudiants Adrien Larbanet et Enguerrand Marcellin ayant suivi cette formation. Ils ont présenté le poster (ci-après) et nous ont communiqué le bref compte rendu ci-dessous :

«Le CETSIS 2008 avait pour thème l'apprentissage par projet ou par une approche différente de la (ou des) matière(s). Ainsi nous, étudiants à I'IUT de Cachan, allions présenter le module «Quadripôles " à l'Université Libre de Bruxelles (ULB) trois jours durant. Ces journées furent partagées entre présentations des POMMADES (POsters, Maquettes, Matériels, DEmonstationS), conférences et tables rondes sur des sujets aussi divers que variés, repas et (nombreuses) pauses café. Notre poster a suscité un certain intérêt chez des représentants d'autres établissements (d'IUT pour la plupart mais aussi de l'ULB). En effet, pour les IUT, le module "Apprendre autrement" était surtout l'occasion de cours de soutien / rappels en électronique, mathématiques, anglais... Les quadripôles nous ont permis de caractériser quelques montages électroniques par le biais d'un nouvel outil mathématique pour nous : les matrices. Rien à voir donc avec un cours de soutien. Nous avons pu goûter à l'hospitalité belge notamment au cours des deux soirées organisées : l'une sur «la plus belle place du monde ", celle de l'hôtel de ville, et l'autre au sein même de l'ULB, dans " la Salle de Marbre". De plus, hors CETSIS, nous avons pu admirer la (magnifique) ville de Bruxelles : l'hôtel de ville donc mais aussi l'Atomium et le Manneken-Pis pour ne nommer que les plus connus. "

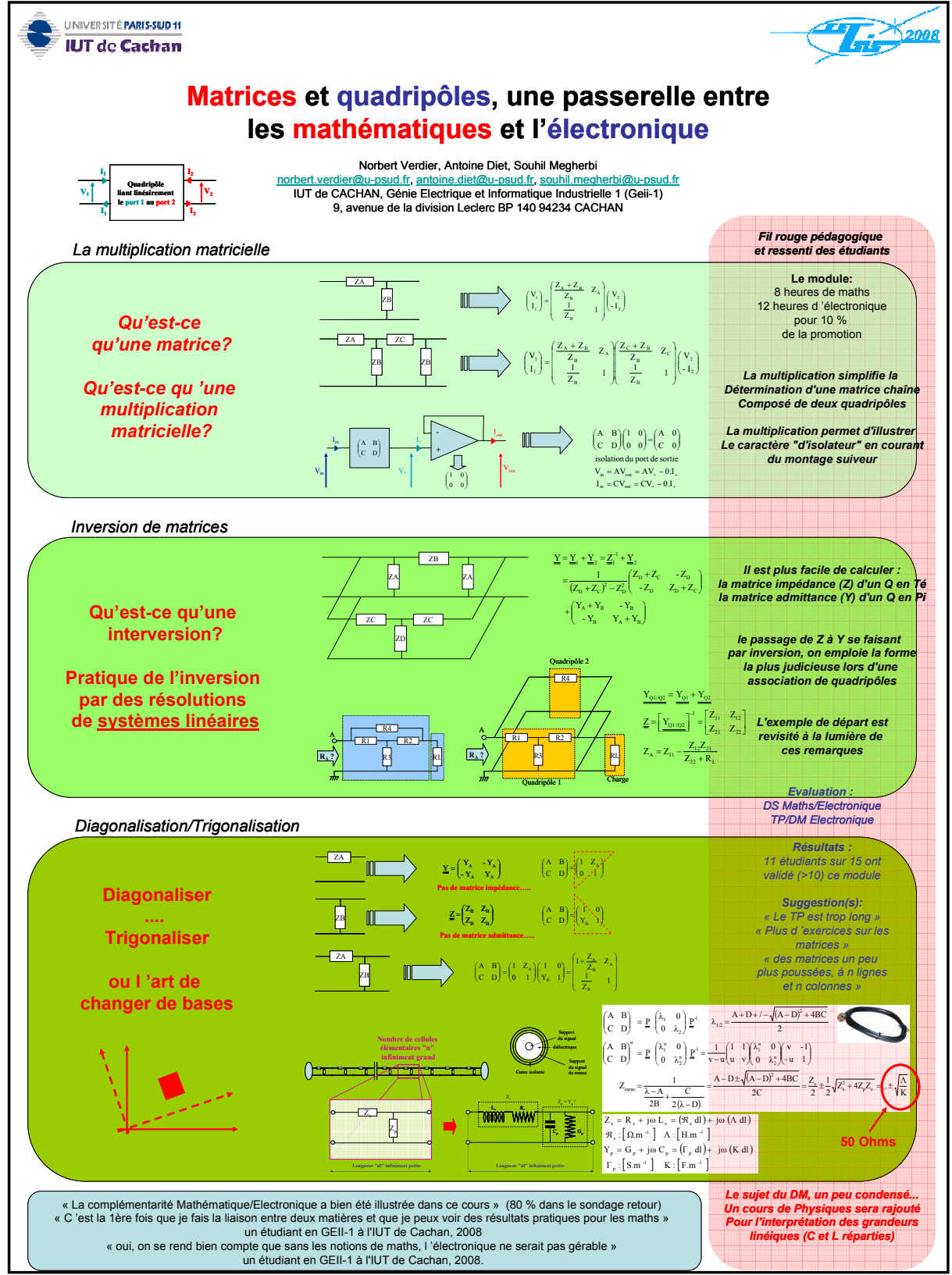

